\title{
Acknowledgments to JINS External Reviewers, 2008
}

\begin{abstract}
The success of JINS as a scientific journal depends on thoughtful, honest, and prompt peer review. Such review would be impossible without the extensive help of colleagues. We wish to acknowledge the following reviewers for manuscripts reviewed between September 1, 2007 and August 31, 2008, with sincere appreciation. Please note that, occasionally, because of the way time frames are figured, a reviewer's name might not appear in this year's list either because s/he was acknowledged previously, or will be thanked in the forthcoming cycle.
\end{abstract}

Acevedo, Amarilis

Adams, Kenneth M.

Adams, Russell L.

Adolphs, Ralph

Aizenstein, Howard

Akshoomoff, Natacha

Alibhai, Shabbir

Allen, Greg

Alpherts, W.C.J.

Altmann, Lori J.

Anderson, Peter John

Andres, Pilar

Andrewes, David

Anger, W. Kent

Anstey, Kaarin Jane

Antonini, Angelo

Antshel, Kevin

Apperly, Ian

Armstrong, Daniel

Arnott, Wendy

Atkinson, Janette

Au, Rhoda

Axelrod, Bradley N.

Aylward, Elizabeth

Balota, David

Banich, Marie T.

Barch, Deanna

Barnea-Goraly, Naama

Barnes, Lisa

Barrett, Anna M.

Barton, Jason

Basso, Demis

Basso, Michael R.

Bastiaanse, Roelien

Bauer, Russell M.

Bearden, Carrie

Bechara, Antoine

Becker, James

Behrman, Andrea

Behrmann, Marlene

Belanger, Heather G.

Belleville, Sylvie

Benedict, Ralph H.B.

Bennett, David A.

Bennett, Pauleen Charmayne

Berg, Raquel

Berger, Andrea
Bernasconi, Andrea

Berndt, Rita S.

Beversdorf, David Q.

Bialystok, Ellen

Bigler, Erin D.

Bilder, Robert M.

Binder, Laurence M.

Bisiacchi, Patrizia

Blackburn, Lynn

Blonder, Lee X.

Boake, Corwin

Bogod, Nicholas M.

Boivin, Michael

Bonan, Isabelle

Bondi, Mark W.

Bornhofen, Cristina

Bouwens, Sharon

Bowden, Stephen C.

Bowers, Dawn

Boyd, Thomas Mitchell

Boyle, Patricia

Bradbury, David A.

Brickman, Adam M.

Brito, Gilberto N.O.

Brown, Franklin C.

Brown, Sandra

Brown, Tanya Maines

Brown, Truman R.

Brugger, Peter

Bucks, Romola Starr

Buizer, Annemieke I.

Burack, Jake

Burdick, Katherine E.

Burgess, Paul W.

Burns, Thomas G.

Buschke, Herman

Butler, Robert W.

Buxbaum, Laurel

Byrd, Desiree

Caccappolo-van Vliet, Elise

Cahill, Lawrence

Caine, Diana

Calkins, Monica E.

Campbell, Meghan

Cappa, Stefano F.

Caramazza, Alfonso

Carlson, Michelle C.
Castellon, Steven A.

Castelo, J. Mimi

Catani, Marco

Cato Jackson, M. Allison

Cavedini, Paolo

Chambers, Chris

Champagne-Lavau, Maud

Chan, Raymond C.K.

Chelune, Gordon J.

Chenery, Helen

Cherbuin, Nicolas

Chicherio, Christian

Christ, Shawn

Christodoulou, Christopher

Cicerone, Keith D.

Cimino, Cynthia R.

Cirino, Paul

Clare, Linda

Clifford, David

Coetzer, Rudi

Cohen, Henri

Collins, Barbara

Colom, Roberto

Comijs, Hannie

Conant, Lisa L.

Conklin, Heather Marie

Constantinou, Marios

Conway, Tim

Cools, Roshan

Copland, David

Corbett, Blythe

Cordoba, Juan

Cosentino, Stephanie

Coslett, H. Branch

Cox, Christiane Sylvia

Craft, Suzanne

Craik, Fergus I.M.

Crane, Paul Kadane

Crawford, John R.

Crowe, Simon F.

Culbertson, Jan L.

Cummings, Jeffrey L.

da Rocha, Felipe Filardi

Dager, Steven

Dalton, Kim

Danckert, James Andrew

Daneman, Meredyth 
Danion, Jean-Marie

Dapretto, Mirella

David, Tony

Davis, Andrew S.

De Jager, Celeste

Decety, Jean

Dede, Duane

Delaney, Dick

Delano-Wood, Lisa

deLeon, Mony

DeLuca, John

Dempster, Frank

Denney, Douglas R.

Dennis, Maureen

Derntl, Birgit

Desposito, Mark

Detterman, Douglas K.

Dewey, Deborah

Dick, Malcolm

Dietens, Erna

Dik, Miranda

Disterhoft, John F.

Doctor, Jason Nicholas

Dogherty, Robert

Donchin, Emanuel

Douglas, Ris

Downing, Marina

Druks, Judit

Duchaine, Brad

Duffy, Charles Joseph

Durgerian, Sally

Durvasula, Ramani

Edmonds, Lisa

Einstein, Gil

Elderkin-Thompson, Virginia

Elias, Merrill F.

Ellenberg, Leah

Ellis, Charles

Ellis, Judy

Engle, Randall

Evans, Jonathan

Ewing-Cobbs, Linda

Exner, Cornelia

Eyler, Lisa T.

Fama, Rosemary

Fantie, Bryan D.

Farace, Elana

Farina, Elisabetta

Farrow, Tom F.D.

Fein, George

Fennell, Eileen B.

Ferman, Tanis J.

Ferraro, Richard

Ferris, Steven

Fillmore, M.T.

Filoteo, J. Vincent

Fimm, Bruno

Fish, Jessica

Fleming, Jennifer

Fletcher, Jack M.

Fordyce, David

Forton, Daniel M.

Foster, Norman L.

Fox, Elaine

Franczak, Malgorzata
Frazier, Thomas William

Friederici, Angela

Friedman, David

Frith, Christopher

Frye, Richard Eugene

Fuentes, Daniel

Gaillard, W.D.

Gale, Shawn D.

Gamst, Anthony

Ganguli, Mary

Garrett, Kelly Davis

Gass, Carlton S.

Gauthier, Isabel

Geerlings, Mirjam

Gepner, Bruno

Gevins, Alan S.

Gilmore, Grover C.

Gioia, Gerard A.

Giovannetti, Tania

Glisky, Elizabeth L.

Glymour, M. Maria

Golan, Ofer

Goldenberg, Georg

Gollan, Tamar H.

Gonzalez, Raul

Gonzalez-Rothi, Leslie J.

Grabowski, Thomas J.

Graff-Radford, Neil

Grande, Laura Jeanne

Grant, Igor

Grant, Steven

Gray, Jeremy

Green, Melissa

Green, Robin

Greher, Michael

Gremley, Shelley

Grisham, Jessica

Grober, Ellen

Grossi, Dario

Grossman, Murray

Gunning-Dixon, Faith

Gunstad, John

Haaland, Vegard Øksendal

Hadjikhani, Nouchine

Haier, Richard J.

Halperin, Jeffrey M.

Hamilton, Joanne M.

Han, S. Duke

Hanks, Robin

Hannay, H. Julia

Harciarek, Michal

Hardan, Antonio

Hart, Carl

Hart, Tessa

Harvey, Danielle

Harvey, Philip D.

Haslam, Catherine

Hassanein, Tarek

Hatta, Takeshi

Haut, Marc W.

Head, Denise

Heaton, Shelley C.

Heffelfinger, Amy K.

Heil, Sarah

Helmstaedter, Christoph
Henry, Julie D.

Hermann, Bruce P.

Hietanen, Jari

High, Walter M.

Hill-Briggs, Felicia

Hillis, Argye

Hilsabeck, Robin C.

Hjaltason, Hauker

Hofer, Scott M.

Holdnack, James Alan

Holtzer, Roee

Horowitz, Todd

Hunter, Scott J.

Huppert, Felicia A.

Hurlemann, René

Ingles, Janet

Ingraham, Loring

Isquith, Peter

Iverson, Grant L.

Ivnik, Robert J.

Jack, Clifford R., Jr.

Jacobson, Sandra W.

Jagust, William

Jahanshahi, M.

Jak, Amy

Jakobson, Lorna Susan

Janowsky, Jeri S.

Jansari, Ashok

Janusz, Jennifer Ann

Jefferson, Angela L.

Jelinek, Lena

Jenkins, Valerie

Jernigan, Terry

Johnson, Julene K.

Johnson, Shannon A.

Johnson, Sterling

Johnson-Greene, Doug

Jones, Rich

Jones, Scott Nelson

Jones-Gotman, Marilyn

Jonkers, Roel

Joseph, Robert

Joyce, Eileen

Kadis, Darren Stefan

Kapczinski, Flavio

Karaivazoglou, Katerina

Kareken, David A.

Karlawish, Jason

Karol, Robert L.

Kaszniak, Alfred W.

Kathmann, Norbert

Katz, Noomi

Kaufmann, Liane

Kaufmann, Walter E.

Kazandjian, Seta

Keilp, John G.

Kemper, Susan

Kennedy, James

Kensinger, Elizabeth

Kerns, Kimberly A.

Kertesz, Andrew

Kiefer, Markus

Kiehl, Kent

King, Tricia Z.

Kingstone, Alan 
Kinsella, Glynda Jane Kirkwood, Michael W. Kirshner, Howard

Klein, Martin

Knight, Robert G.

Koelman, J.H.T.M.

Konrad, Kerstin

Koop, Jennifer

Kopelman, Michael D.

Kotz, Sonja

Kranczioch, Conny

Kremen, William S.

Kryspin-Exner, Ilse

Kuntsi, Jonna

Kuslansky, Gail

Laatsch, Linda

Lacy, Maureen

Lamar, Melissa

Langdon, Robyn

Lange, Rael T.

Lavoie, Marc E.

Laws, Keith R.

Leblanc, Erin

Lee, Dongwook

Lee, Gregory Price

Lee, Joseph H.

Lemogne, Cedric

Leplow, Bernd

Letendre, Scott

Leveroni, Catherine

Levin, Harvey S.

Levine, Brian

Lewine, Jeffrey David

Lewis, Michael

Libon, David J.

Licht, Robert

Lillywhite, Leasha M.

Lincoln, Alan J.

Lineweaver, Tara

Liossi, Christina

Loewenstein, David

Lopez, Oscar L.

Loring, David W.

Lowenstein, David

$\mathrm{Lu}$, Lisa $\mathrm{H}$.

$\mathrm{Lu}, \mathrm{Po} \mathrm{H}$.

Lucas, John A.

Luis, Cheryl

Lyytinen, Heikki Juhani

MacDonald, Stuart

Mahone, Mark

Maki, Pauline M.

Malapani, Chara

Malec, James F.

Malloy, Paul F.

Man, David W.K.

Manly, Tom

Marcopulos, Bernice A.

Marcotte, Ann C.

Marcotte, Thomas D.

Mark, Victor W.

Marsiske, Michael

Marson, Daniel C.

Martens, Sander

Martin, David John
Martin, Roy C.

Maruff, Paul

Mateer, Catherine A.

Matsui, Mie

Mattingley, Jason

Mattson, Sarah N.

Max, Jeffrey E.

McAllister, Thomas W.

McAndrews, Mary Pat

McCrea, Michael

McCrea, Simon M.

McDaniel, Mark

McDonald, Carrie R.

McDowd, Joan

McGurk, Susan Regina

McMonagle, Paul

Meador, Kimford J.

Mendez, Mario

Mennemeier, Mark S.

Menor, Julio

Meyerhoff, Dieter

Meyers, Christina A.

Milders, Maarten

Millar, Keith

Millis, Scott R.

Milne, Elizabeth

Moberg, Paul J.

Moceri, Victoria

Monterosso, John

Moore, Anna Bacon

Moran, Maria

Morgan, Joel E.

Morgello, Susan

Moritz, Steffen

Morris, Robin G.

Mortimer, James A.

Mostofsky, Stewart H.

Mottron, Laurent

Müller, Ralph-Axel

Multhaup, Kristi

Mungas, Dan

Munro, Cynthia A.

Murphy, Declan

Murphy, Jennifer

Na, Duk L.

Nass, Ruth D.

Nebes, Robert D.

Nelles, Gereon

Nelson, Nathaniel William

Nester, Michael J.

Newman, Mary C.

Nicholls, Mike

Nichols, Sharon

Nixon, Sara Jo

Novack, Thomas A.

O'Bryant, Sid

O'Carroll, R.E.

Ochipa, Cynthia

Oerbeck, Beate

Ogden, Jenni A.

O'Sullivan, M.

Ownsworth, Tamara

Ozonoff, Sally J.

Palmer, Barton W.

Pardo, Jose V.
Park, Sohee

Parker, Elizabeth Stewart

Parmenter, Brett A.

Parsons, Thomas D.

Paul, Robert

Pavone, Piero

Pedraza, Otto

Peery, Shelley

Pell, Marc D.

Pennington, Bruce Franklin

Perdices, Michael

Perlstein, William Michael

Perry, William

Petersson, Karl Magnus

Piek, Jan Patricia

Pieters, Guido

Piguet, Olivier

Pohlmann-Eden, B.

Ponton, Marcel O.

Powell, Thomas

Prigatano, George P.

Rabkin, Judith

Rakitin, Brian C.

Rampacher, Friederike

Randolph, Christopher

Rankin, Katherine

Raskin, Sarah

Razani, Jill

Reed, Bruce R.

Reid, Wayne Gordon Joseph

Reilly, Jamie

Rich, Jill B.B.

Richard, Brown

Richards, Marcus

Riley, Gerard Anthony

Rimrodt, Sheryl L.

Ris, M. Douglas

Risse, Gail L.

Ritchie, Karen

Rivera Mindt, Monica

Rivera, Susan

Roberts, John

Roberts, Katherine Leonie

Robertson, Ian

Robinson, Robert G.

Roebuck Spencer, Tresa

Roriz-Cruz, Metheus

Rosano, Caterina

Rosen, Allyson

Ross, Elliott D.

Rosselli, Monica

Roth, Robert M.

Ryan, Christopher

Ryan, Joseph J.

Sabsevitz, David

Salmon, David

Salo, Ruth

Salthouse, Timothy A.

Samson, Dana

Sano, Mary

Savage, Cary

Savage, Greg

Scarmeas, Nikolaos

Schagen, S.B.

Schatz, Jeffrey C. 
Scherwath, A.

Schlund, Michael W.

Scholten, Marion

Schultz, Robert

Schuurman, P.R.

Schweinsburg, Alecia

Schweizer, Karl

Seidenberg, Michael

Selnes, Ola A.

Sharland, Michael

Sherer, Mark

Shimamura, Arthur

Shum, David

Shur, Syvan

Siedlecki, Karen L.

Sinha, Pawan

Slick, Daniel Joseph

Slomine, Beth S.

Small, Brent J.

Smeding, H.M.M.

Smith, Gwenn

Soldan, Anja

Spitzer, Manfred

Stebbins, Glenn T.

Stern, Hal

Story, Tyler

Strange, Bryan

Strauss, Milton

Stroup, Lisa

Stuss, Donald T.

Suhr, Julie

Summers, Mathew James
Suslow, Thomas

Swanson, Sara J.

Sweet, Jerry J.

Tager-Flusberg, Helen

Tanaka, James

Tannock, Rosemary

Tapert, Susan Frances

Tate, Robyn

Tchanturia, Kate Ketevan

Teng, Edmond

Teresi, Jeanne

Thoma, Robert J.

Thornton, Allen E.

Tierney, Mary

Tieu, Kinh

Tomer, Rachel

Tracy, Joseph Igoe

Tremont, Geoffrey

Trivedi, Mehul Alkesh

Trojano, Luigi

Troyer, Angela K.

Tse, Chi-Shing

Tupper, David E.

Turkstra, Lyn

Turnbull, Oliver

Twamley, Elizabeth W.

Vallar, Giuseppe

van Belle, Gerald

Van der Elst, Wim

Vanderploeg, Rodney D.

Vandersluis, S.

VanGorp, Wilfred
Vaskinn, Anja

Vemuri, Prashanthi

Verghese, Joe

Vinogradov, Sophia

Vivas, Leticia

Waber, Deborah P.

Wadley, Virginia G.

Wefel, Jeffrey S.

Weller, Joshua

West, Robert

Whalley, L.

Wheaton, Lewis

White, Desiree

White, Keith

Widaman, Keith

Wills, Karen

Wilson, Robert S.

Wishart, Heather A.

Woodard, John L.

Woods, Steven Paul

$\mathrm{Wu}$, Samuel

Yaffe, Kristine

Yang, Frances

Yelnik, Alain

Yochim, Brian P.

Yurgelun-Todd, Deborah

Zaitchik, Deborah

Zalonis, Ioannis

Zatorre, Robert J.

Zelko, Frank

Zimmerman, Molly

Zola, Stuart 